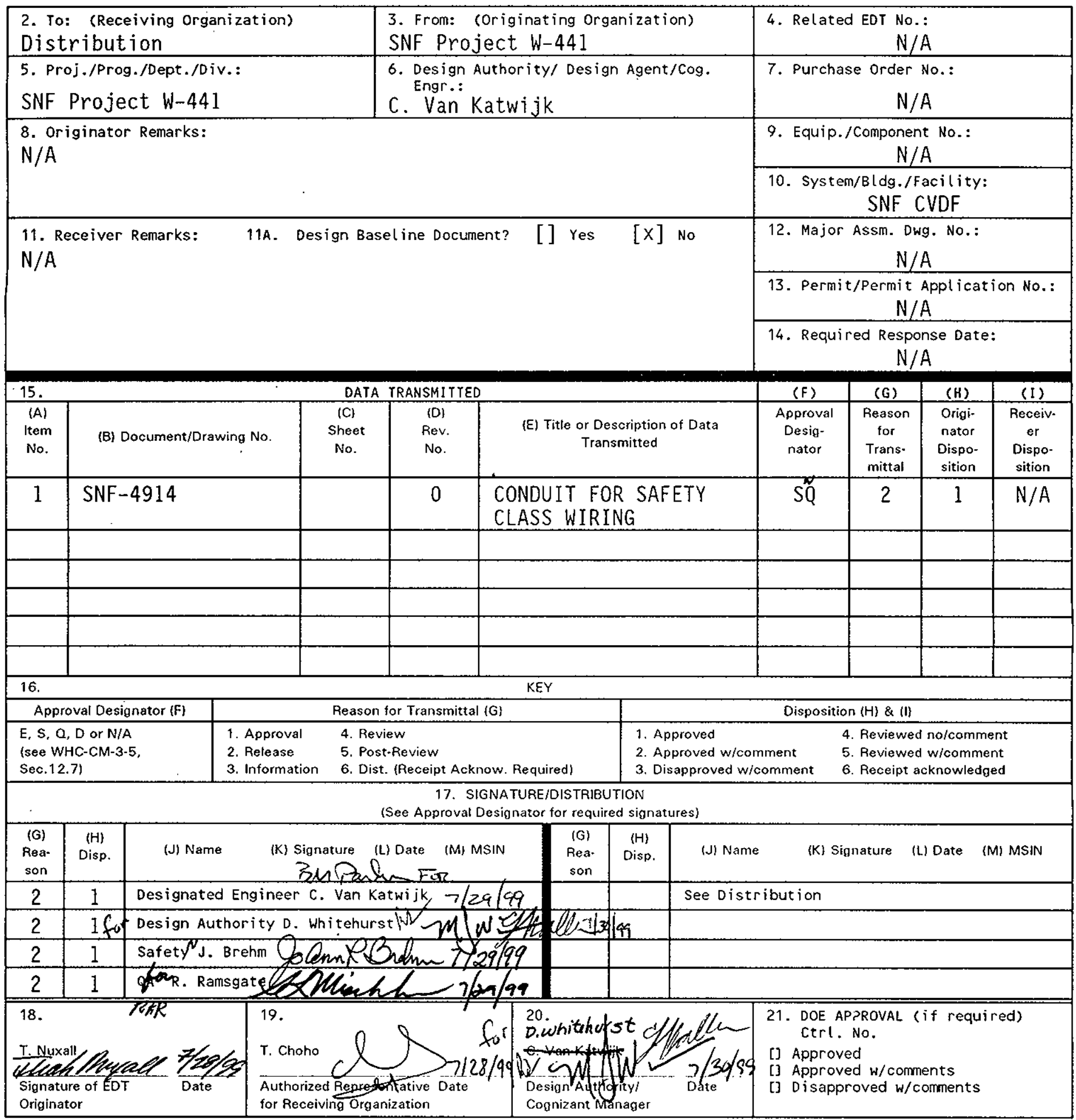

$\mathrm{BD}-7400-172-2(05 / 96)$ GEF097 


\title{
CONDUIT FOR SAFETY CLASS WIRING
}

\author{
Carl Van Katwijk
}

Numatec Hanford, Richland, WA 99352

U.S. Department of Energy Contract DE-AC06-96RL13200

EDT/ECN: 626324

Org Code: $2 \mathrm{G} 300$

B\&R Code: 39EW40400

UC: 620

Charge Code: $105559 / \mathrm{A} 000$

Total Pages: 34

Key Words: Conduit for Safety Class Wiring

Abstract: Conduit for Safety Class Wiring

TRADEMARK DISCLAIMER. Reference herein to any specific commercial product. process, or service by trade name, trademark. manufacturer, or othervise, does not necessarily constitute or imply its endorsement. secommendation, or favoring by the United States Government or any agency there of or its contractors or sulhcontractors.

Printed in the United States of America. To obtain copies of this document, contact: Document Control Scrvices. P.O. Box 950. Mailstop H6-08, Richland WA 99352. Phone (509) 372-2420: Fax (509) 376-4989.

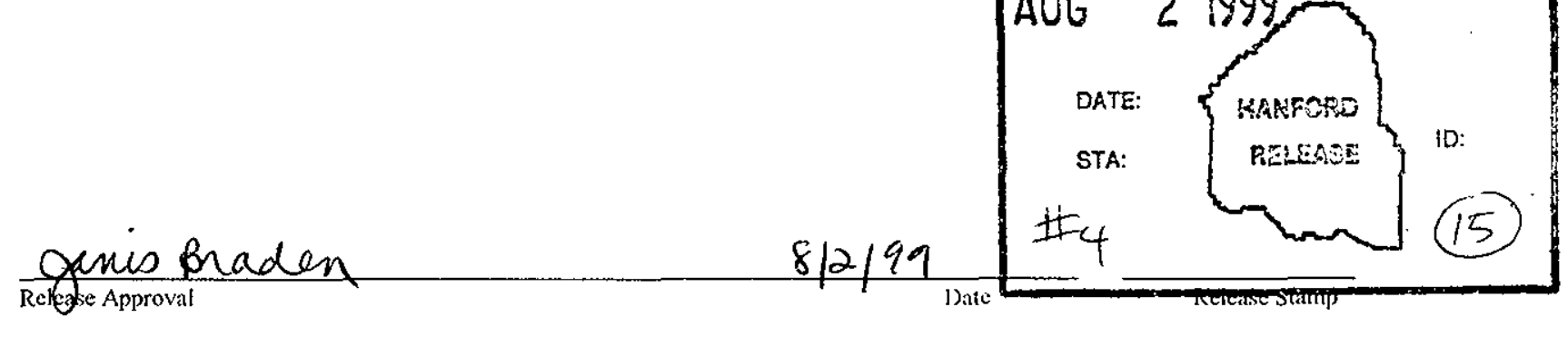

Approved for Public Release 


\begin{tabular}{|l|l|}
\hline Commercial Grade Item Upgrade Dedication Form & SNI-4914. Rev. 0 \\
\hline $\begin{array}{l}\text { ECN No. NA CGI No. CGI-SNF-D-93-2-C1-052 } \\
\text { Title: CONDUIT FOR SAFETY CLASS WIRING }\end{array}$ & Page l of 33 \\
\hline
\end{tabular}

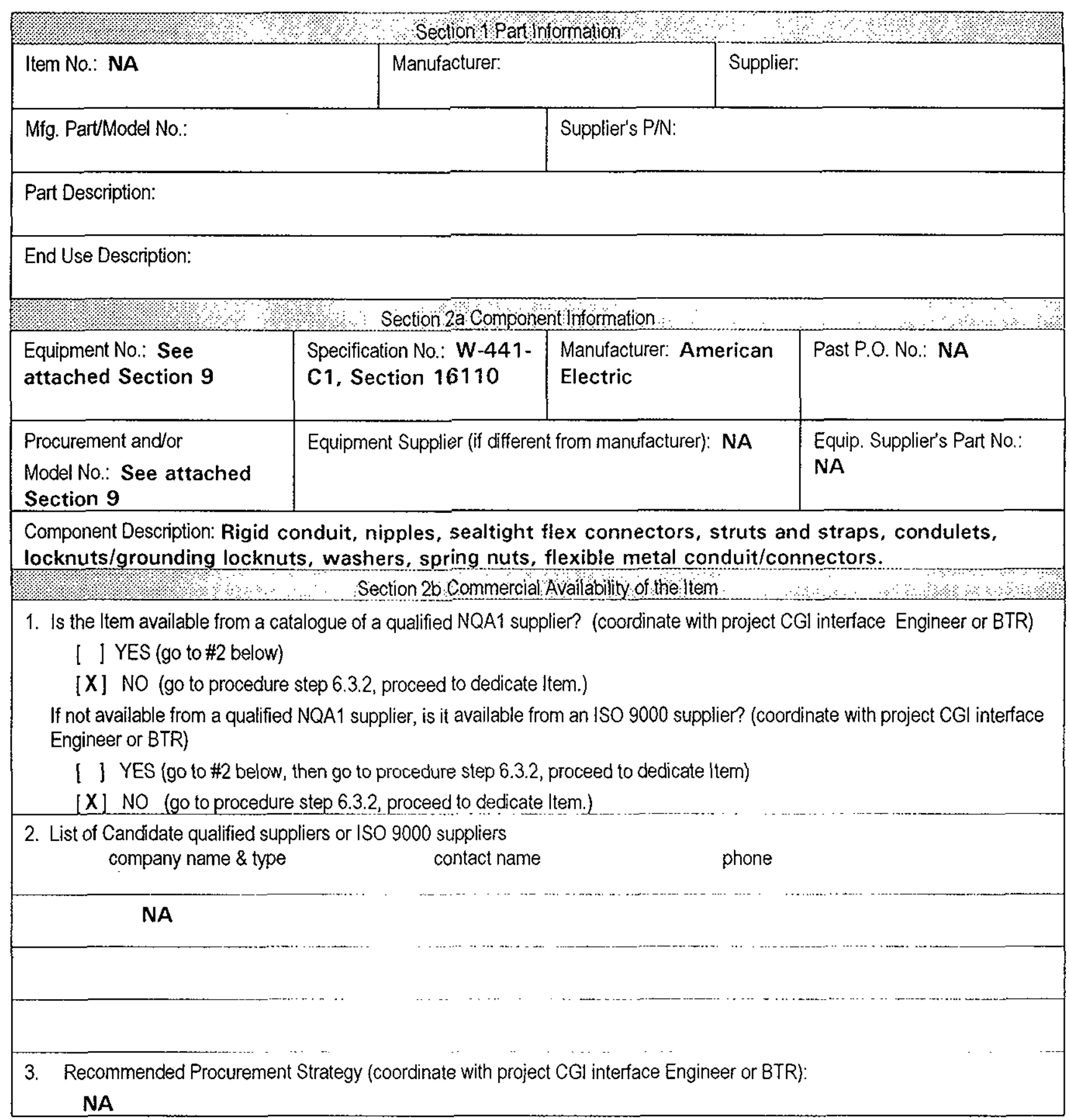




\begin{tabular}{|l|l|}
\hline Commercial Grade Item Upgrade Dedication Form & SNF.4914. Rev. 0 \\
\hline $\begin{array}{l}\text { ECN No. NA CGI No. CGI-SNF-D-93-2-C1-052 } \\
\text { Titte: } \text { CONDUIT FOR SAFETY CLASS WIRING }\end{array}$ & Page 2 of 33 \\
\hline
\end{tabular}

Section 2c CGI Determination

1. Question \#1: is the Item subject to design or specification requirements that are unique to nuclear facilities or activities?

1 ] YES (the Item is not commercial grade)

[X] NO (continue)

2. Question \#2: is the Item used in applications other than nuclear facilities or activities?

[ ] NO (the item is not commercial grade)

[X] YES (continue)

3. Question \#3: Is the Item ordered from manufacturer/supplier on the basis or specifications set forth in the published product information (e.g. manufacturer's catalog)?

( ) NO (the ltem is not commercial grade)

[X] YES (continue)

[X] All three criteria have been satisfied. The Item meets the definition of commercial grade.

Section $2 \mathrm{~d}$ Reason for Dedication.

The above described tem is being Dedicated for use in the application cited for the following reason(s)

[X] Item is being purchased from a non ESL manufacturer supplier as commercial grade to be used in a Safety Class application.

[ ] Item is being purchased from a non ESL manufacturer supplier as commercial grade to be used in a Safety Significant application.

1 1 Item was purchased from a non ESL manufacturer supplier as commercial grade to be used in a Safety Class application.

[ ] Item was purchased from a non ESL manufacturer supplier as commercial grade to be used in a Safety Significant application.

1 1 Other ('like-for-like', similar, substitution, replacement evaluation)

A. Par//Component Safety Function:

Section 3 Failure Effects Evaluation

1. Provide conduit protection, structural support for safety class wiring.

2. Maintain critical function before, during, and after Seismic Event.

3.

B. Part/Component Functional Mode:

Safety Function \#1:

[ ] Active - Mechanical or Electrical change of state is required to occur for the component to perform its safety function

[X] Passive - Change of state is not required for the component to perform its safety function

Safety Function \#2:

[ ] Active -Mechanical or Electrical change of state is required to occur for the component to perform its safety function.

[X] Passive - Change of state is not required for the component to perform its safety function

Safety Function \#3:

1 ] Active - Mechanical or Electrical change of state is required to occur for the component to perform its safety function.

1 ] Passive - Change of state is not required for the component to perform its safety function 


\begin{tabular}{|l|l|}
\hline Commercial Grade Item Upgrade Dedication Form & SNI-4914. Rev. 0 \\
\hline $\begin{array}{l}\text { ECN No. NA CGI No. CGI-SNF-D-93-2-C1-052 } \\
\text { Title: CONDUIT FOR SAFETY CLASS WIRING }\end{array}$ & Page 3 of 33 \\
\hline
\end{tabular}

C. Host Component Safety Function (if applicable): NA

1.

D. Failure Mode(s) and the effects on component or system safety function (see Worksheet 1):

1. Fracture or material fatigue leads to loss of structural support and possible damage to wiring.

2. Loss of sealtight integrity of fittings could result in moisture corrosion and possible damage to wiring.

3.

4.

5.

Section 4 Environmental \& Natural Phenomena Hazard Design

Environmental Qualification Required:

If yes: Environmental Qualification Requirements

Yes [ ]

Limiting Environmental Conditions:

No $[\mathbf{X}]$

Required Safety Functions:

Qualification Period:

Environmental Condition B

If yes: NPH Design Requirements

Natural Phenomena Hazard (NPH) Design Required:

Yes [X]

Performance Category: PC-3

No [ ]

NPH Design Req'ts.: Seismic Condition C

HNF.PRO-97, Rev. 0

Required Safety Functions: Provide protection and support for safety class wiring. Maintain critical function before, during, and aftei Seismic Event.

Section 5 Component Functional Classification

[X] Safety Class (SC) [ | General Service (GS) | ] Safety Significant (SS)

If part/component classification is different from host component/system, document basis. NA

$\frac{\text { Section 6 (Reserved) }}{\text { Section 7 (Reserved) }}$

.. . $.1 .1 \%$ Section 8 References (for Functional Classification)

National Codes/Standards: IEEE

628

Safety Analysis Report (SAR):

HNF-SD-SNF-SAR-002,

Drawings: HNF-SD-SNF-SEL-002,

Rev. 6A

Rev. 4A

Vendor Manual/Manufacturer/Supplier Information: Rigid Conduit - LTV Steel Tubular Products/Galvite

Rigid Conduit Nipples/Connectors /Locknuts/Beam Clamps/Reducers-Appleton Catalog CF-2 Pages 4, 5, 6, 7, 10;

Covers and gaskets for Form 35 Conduit Outlet Boxes - Appleton Catalog A-8, Pages 6 and 7

Conduit Nipples - Conduit Pipe Products Catalog Page KG

Flexable Steel Conduit - Thomas \& Betts Liquidtight Flexible Metal Conduit and Fittings/Connectors,

Pages 8 and 23.

Metal Framing Channels/Pipe Straps - Superstrut Series 1000 Section 1, Pages 6, 7, 39;

Fender Washers - ABC Fasteners Page 2-1;

PS-146 Continuous Threaded Rod/Clamping Nut with spring - Power Strut Pages 40, 42 


\begin{tabular}{|l|l|}
\hline Commercial Grade Item Upgrade Dedication Form & SNF-4914. Rev. 0 \\
\hline ECN No. NA $\quad$ CGI No. CGI-SNF-D-93-2-C1-052 & Page 4 of 3.3 \\
Title: CONDUIT FOR SAFETY CLASS WIRING & \\
\hline
\end{tabular}

Other:

Section 9 Critical Characteristics.

\begin{tabular}{|l|c|c|c|c|}
\hline Critical Characteristics Verification & Acceptance Criteria/Tolerances & Acceplance \\
Method & ID & Funclion \\
Document: American Electric & & & & \\
Purchase Orders & & & \\
C188R12TM3157 6/16/99; & & & \\
C188R12TM3158, 6/16/99 and & & & & \\
C188R12TM3168, 6/17/99 & & & \\
HNF-SD-SNF-SEL-002, Rev. 6A & & & & \\
\hline
\end{tabular}

1. Item Identification Critical Characteristics (necessary for reasonable assurance that the Item delivered is the Item specified)

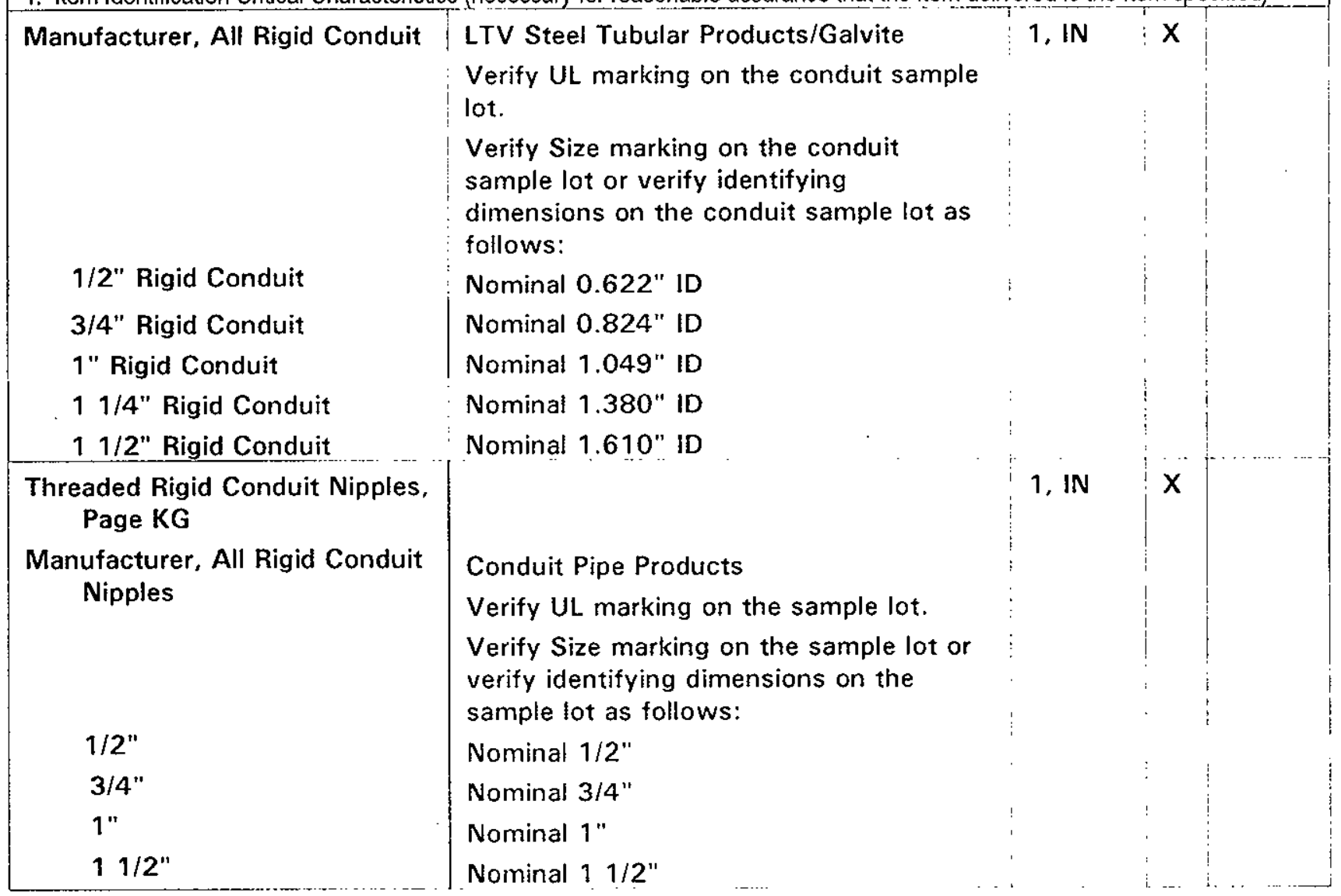




\begin{tabular}{|l|l|}
\hline Commercial Grade Item Upgrade Dedication Form & SNF-4914. Rev. 0 \\
\hline ECN No. NA CGI No. CGI-SNF-D-93-2-C1-052 & Page 5 of 33 \\
Title: CONDUIT FOR SAFETY CLASS WIRING & \\
\hline
\end{tabular}

Threaded Rigid Conduit Bushed Nipples, Page 6

Manufacturer, All Rigid Conduit Bushed Nipples

CN-50

CN-75

$\mathrm{CN}-100$

$\mathrm{CN}-125$

CN-150

Threaded Rigid Conduit, Connectors, Page 5 Manufacturer, All Threaded Rigid Conduit 3 Piece Connectors

EC-50

EC-75

EC-100

EC-125

EC-150
Appleton Electric Company

Verify UL marking on the sample lot. Verify part identifying marking on the sample lot or verify identifying Dimensions on the sample lot as follows: Nominal 1/2" -"A" Dimension, Nominal $7 / 16^{\prime \prime}$

Nominal 3/4" -"A" Dimension, Nominal $1 / 2^{\prime \prime}$

Nominal 1" -"A" Dimension, Nominal 5/8"

Nominal $11 / 4 "$-"A" Dimension, Nominal $11 / 16^{\prime \prime}$

Nominal $11 / 2$ " -A" Dimension, Nominal $7 / 8 "$

1, IN

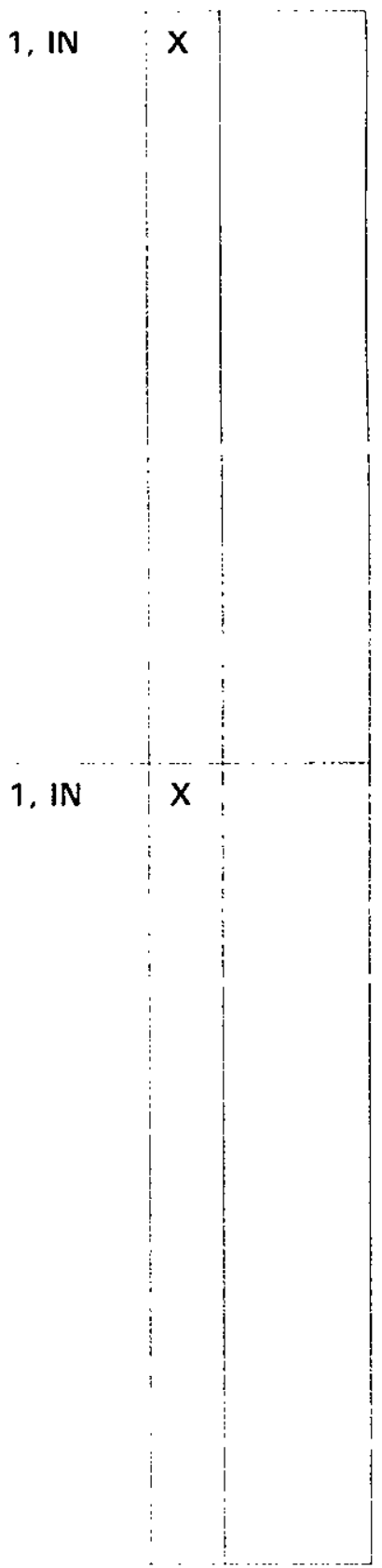




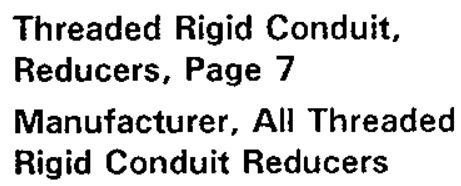

RB75-50

RB100-50

RB150-50

RB100-75

RB150-75

RB125-100

RB150-100

Rigid Conduit Condulet, Form 35, Page 6

Manufacturer, All Rigid Conduit Condulet Form 35

LB/T/LR/LL- $1 / 2^{\prime \prime}$

LB/T/LR/LL-3/4"

LB/T/LR/LL-1"

LB/T/LR/LL-1 1/2"

Rigid Conduit Locknuts, Page 10

Manufacturer, All Rigid Conduit Locknuts / Grounding Locknuts
BL-50 / GL-50
BL-75 / GL-75
BL-100/GL-100
BL-125 / GL-125
BL-150/GL-150

\section{Appleton Electric Company}

Verify UL marking on the sample lot.

Verify identifying marking on the parts in the sample lot or verify identifying dimension on the sample lot as follows:

$3 / 4^{\prime \prime}-1 / 2^{\prime \prime}$

$1 "-1 / 2 "$

$11 / 2^{\prime \prime}-1 / 2^{\prime \prime}$

$1 "-3 / 4 "$

$11 / 2 "-3 / 4 "$

$11 / 4^{\prime \prime}-1 "$

$11 / 2 " 1 "$

Appleton Electric Company

Verify UL marking on the sample lot.

Verify Size marking on the condulet sample lot or verify identifying dimension on the condulet sample lot as follows:

Nominal $1 / 2^{\prime \prime}$

Nominal 3/4"

Nominal $1 "$

Nominal 1 1/2"

Appleton Electric Company

Verify UL marking on the sample lot.

Verify Size marking on the locknut sample tot or verify identifying dimension on the locknut sample lot as follows:

Nominal $1 / 2^{\prime \prime}$

Nominal 3/4"

Nominal 1 "

Nominal $11 / 4^{\prime \prime}$

Nominal $11 / 2^{\prime \prime}$

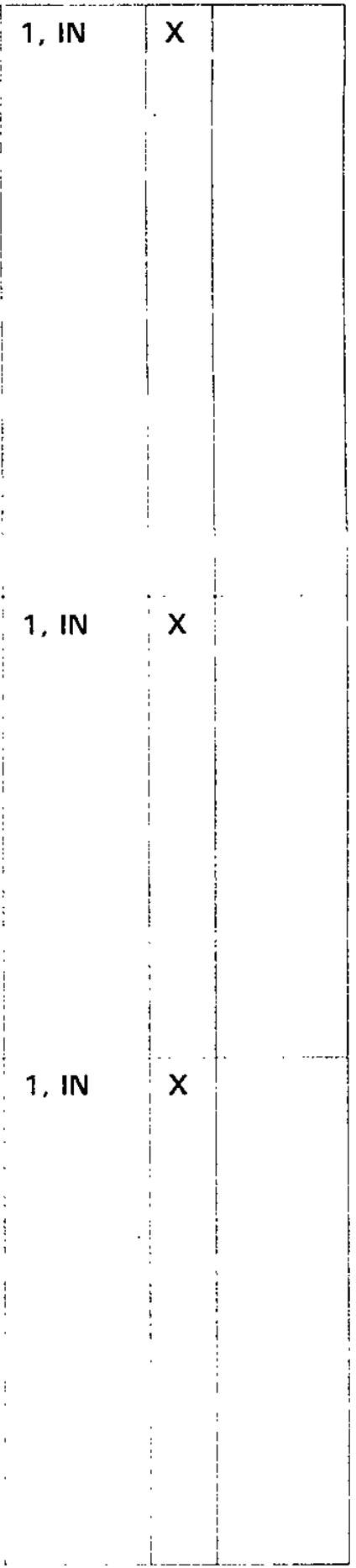




\begin{tabular}{|l|l|}
\hline Commercial Grade Item Upgrade Dedication Form & SN1:4914. Rev. 0 \\
\hline $\begin{array}{l}\text { ECN No. NA CGI No. CGI-SNF-D-93-2-C1-052 } \\
\text { Title: CONDUXT FOR SAFETY CLASS WIRING }\end{array}$ & Page 7 of 3.3 \\
\hline
\end{tabular}

\section{Rigid Metal Conduit Beam Clamps, Page 4 \\ Manufacturer, All Rigid Metal \\ Conduit Beam Clamps}

BH-502

BH-503

\section{Liquidtight Flexible Steel \\ Conduit, Page 8 \\ Manufacturer, All Flexible \\ Conduit}

\section{Type LA-11}

Type LA-12

Type LA-13

Type LA-14

Type LA-15

Liquidtight Flexible Metal Conduit Fittings, Page 23

Manufacturer, Flexible Conduit Connectors

5332

5333

5334

5335

5336
Appleton Electric Company

Verify identifying marking on the sample lot or verify identifying dimensions on the sample lot as follows:

2" Size, Jaw Opening Nominal 15/16"

$21 / 2$ " Size, Jaw Opening Nominal 1 $1 / 16^{\prime \prime}$

Thomas \& Betts

Verify UL marking on the conduit sample lot.

Verify Size marking on the conduit sample lot or verify identifying dimensions on the conduit sample lot as follows:

Nominal $1 / 2$ " Diameter

Nominal 3/4" Diameter

Nominal 1" Diameter

Nominal $11 / 4$ " Diameter

Nominal $11 / 2$ " Diameter

Thomas and Betts

Verify UL marking on the sample lot.

Verify identifying marking on the sample lot or verify identifying dimensions on the sample lot as follows:

Nominal 1/2" -"A" Dimension, Nominal 1 $3 / 8 "$

Nominal 3/4" ."A" Dimension, Nominal 1 21/32"

Nominal 1" -"A" Dimension, Nominal 1 7/8"

Nominal $11 / 4 "$ " A" Dimension, Nominal 2 9/32"

Nominal 1 1/2" -"A" Dimension, Nominal $223 / 32 "$

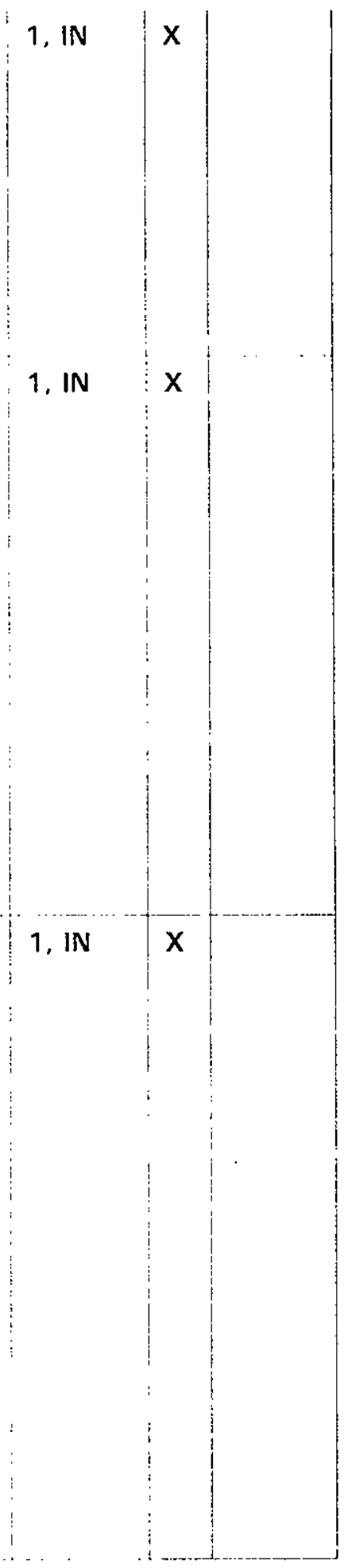


Metal Framing Channels/Pipe Straps, Pages 7 and 39

Manufacturer, All Framing

Channels/Pipe Straps

Half Slot Channel

$$
\text { A-1200-HS }
$$

702 Pipe Straps

$$
702-1 / 2
$$

$702-3 / 4$

702-1

702-1 $1 / 4$

702-1 $1 / 2$

Fender Washer, Page 2-1. Manufacturer

20004

20008

20015

Fasteners, Pages 40 and 42

Manufacturer

PS 146 Continuous

Threaded Rod

PS RS Clamping Nut with Regular Spring.

PS RS
Superstrut

Nominal $15 / 8 "$ x $15 / 8^{\prime \prime}, 12$ gage.

Verify identifying marking on the sample lot or verify identifying dimensions on the sample lot as follows:

Pipe OD Nominal 0.840", 14 gage

Pipe OD Nominal 1.050", 14 gage

Pipe OD Nominal 1.315", 14 gage

Pipe OD Nominal 1.660", 14 gage

Pipe OD Nominal 1.900", 14 gage

$A B C$ Fasteners

$1 / 4^{\prime \prime} \times 11 / 4^{\prime \prime}$

$3 / 8^{\prime \prime} \times 11 / 2^{\prime \prime}$

$1 / 2 " \times 2 "$

Powerstrut

Nominal 3/8"

Nominal $1 / 2^{\prime \prime}$

Nominal 5/8"

Nominal 3/4"

$1 / 4^{\prime \prime} \times 20$

$3 / 8^{\prime \prime} \times 16$

$1 / 2^{\prime \prime} \times 13$

$5 / 8^{\prime \prime} \times 11$

$3 / 4 " \times 10$

$7 / 8^{\prime \prime} \times 9$
1, IN

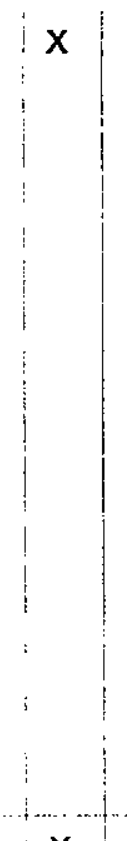

1, IN

$\mathrm{X}$

$1, \mathrm{IN}$
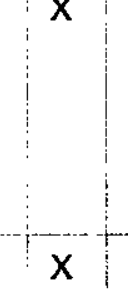

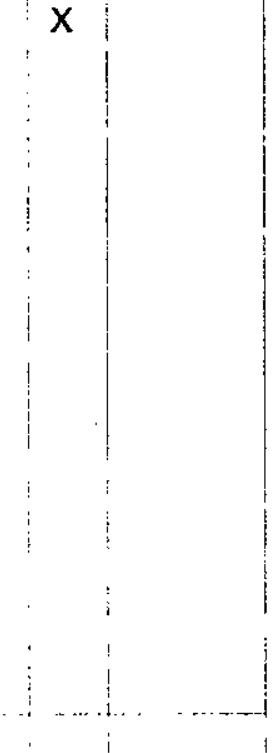

\begin{tabular}{|c|c|c|c|}
\hline All Size Rigid Conduit, Material & Steel (Note 3) & $\begin{array}{l}1, \mathbb{I N} \\
1, T\end{array}$ & $\mathrm{x}$ \\
\hline $\begin{array}{l}\text { All Size Rigid Conduit Nipples, } \\
\text { Material }\end{array}$ & Steel (Note 3) & $\begin{array}{l}1, \mathrm{IN} \\
1, \mathrm{~T}\end{array}$ & $x$ \\
\hline $\begin{array}{l}\text { All Size Threaded Rigid Conduit } \\
\text { Bushed Nipples, Material }\end{array}$ & Steel (Note 3) & $\begin{array}{l}1, \text { IN } \\
1, T\end{array}$ & $x$ \\
\hline
\end{tabular}

2. Physical Critical Characteristics (for reasonable assurance that the Item delivered is the Item specified) 


\begin{tabular}{|l|l|}
\hline Commercial Grade Item Upgrade Dedication Form & SNF-4914. Rev. 0 \\
\hline ECN No. NA & Page 9 of 33 \\
Title: CONDUIT FOR SAFETY CLASS WIRING & \\
\hline
\end{tabular}

\begin{tabular}{|c|c|c|c|c|}
\hline $\begin{array}{l}\text { Threaded Rigid Conduit, Three } \\
\text { Piece Connectors, Material }\end{array}$ & Steel (Note 3) & $\begin{array}{l}1, \text { IN } \\
1, T\end{array}$ & $x$ & \\
\hline $\begin{array}{l}\text { Threaded Rigid Conduit, } \\
\text { Reducers, Material }\end{array}$ & Steel (Note 3) & $\begin{array}{l}1, \text { IN } \\
1, T\end{array}$ & $x$ & \\
\hline $\begin{array}{l}\text { Rigid Conduit Condulet Form } \\
\text { 35, Material }\end{array}$ & Steel (Note 3) & $\begin{array}{l}1, \mathrm{IN} \\
1, \mathrm{~T}\end{array}$ & $x$ & \\
\hline $\begin{array}{l}\text { Rigid Conduit, } \\
\text { Locknuts/Grounding Locknuts, } \\
\text { Material }\end{array}$ & Steel (Note 3) & $\begin{array}{l}1, \mathrm{IN} \\
1, \mathrm{~T}\end{array}$ & $x$ & \\
\hline $\begin{array}{l}\text { Rigid Metal Conduit, Beam } \\
\text { Clamps, Material }\end{array}$ & Steel (Note 3) & $\begin{array}{l}1,1 \mathrm{~N} \\
1, \mathrm{~T}\end{array}$ & $\mathrm{x}$ & \\
\hline $\begin{array}{l}\text { All Size Liquidtight Flexible } \\
\text { Steel Conduit, Jacketing } \\
\text { Material }\end{array}$ & Flexible PVC Compound (Note 3) & $\begin{array}{l}1, \text { IN } \\
1, T\end{array}$ & $x$ & \\
\hline $\begin{array}{l}\text { Flexible Steel Conduit, } \\
\text { Connectors, Material }\end{array}$ & Steel (Note 3) & $\begin{array}{l}1, \mathrm{IN} \\
1, \mathrm{~T} \\
\end{array}$ & $x$ & \\
\hline Framing Channel, Material & Steel (Note 3) & $\begin{array}{l}1, \mathrm{IN} \\
1, \mathrm{~T} \\
\end{array}$ & $x$ & \\
\hline $\begin{array}{l}\text { Framing Channel } 702 \text { Pipe } \\
\text { Straps, Material }\end{array}$ & Steel (Note 3) & $\begin{array}{l}1, \mathrm{IN} \\
1, \mathrm{~T}\end{array}$ & $x$ & \\
\hline Fender Washer, Material & Zinc Coated Steel (Note 3) & $\begin{array}{l}1, \text { IN } \\
1, T\end{array}$ & $x$ & \\
\hline $\begin{array}{l}\text { Fasteners, } \\
\text { PS } 146 \text { Continuous } \\
\text { Threaded Rod. } \\
\text { PS RS Clamping Nut with } \\
\text { Regular Spring. }\end{array}$ & $\begin{array}{l}\text { Steel (Note 3) } \\
\text { Steel (Note 3) }\end{array}$ & $\begin{array}{l}1, \mathrm{IN} \\
1, \mathrm{~T}\end{array}$ & $x$ & \\
\hline & & & & \\
\hline & & & & \\
\hline 3. Performance Critical Characteristics & reasonable assurance that & & & \\
\hline Environmental & Note 1 & & & \\
\hline Seismic Condition C & Note 2 & $\begin{array}{l}1, T \\
1, A \\
\end{array}$ & & $x$ \\
\hline
\end{tabular}




\begin{tabular}{|l|l|}
\hline Commercial Grade Item Upgrade Dedication Form & SNF-4914. Rer. () \\
\hline $\begin{array}{l}\text { ECN No. NA } \\
\text { Titie: CONDUIT FOR SAFETY CLASS WIRING }\end{array}$ & Page 10 of 33 \\
\hline
\end{tabular}

4. Notes and Legend:

1. The components are not subject to degradation from the $40^{\circ} \mathrm{F}$ or $60 \% \mathrm{RH}$ or $115^{\circ} \mathrm{F}$ and $22 \% \mathrm{RH}$ conditions and are suitable for Environmental Condition B application.

2. Maintain critical function before, during, and after Seismic event. Equipment that has been shaker-table tested should not be installed in a plant (Ref. IEEE Standard 344-1984, Section 7). Consequently, the seismic test constitutes a destructive test. Seismic Design may be verified by analyis of the installed conduit system.
Acceptance Method:

1. Special Test and Inspection

1, IN for Inspection

1, T for Test

1,A for Acceptance by Analysis

2. Commercial Grade Survey

3. Source Verification

4. Vendor/Item History

3. Material verification acceptance method may be by either inspection or test.

Approvals:
Designated Engineer:
Design Authority:
QA Engineer: Section 10 Initial Review:and Approval




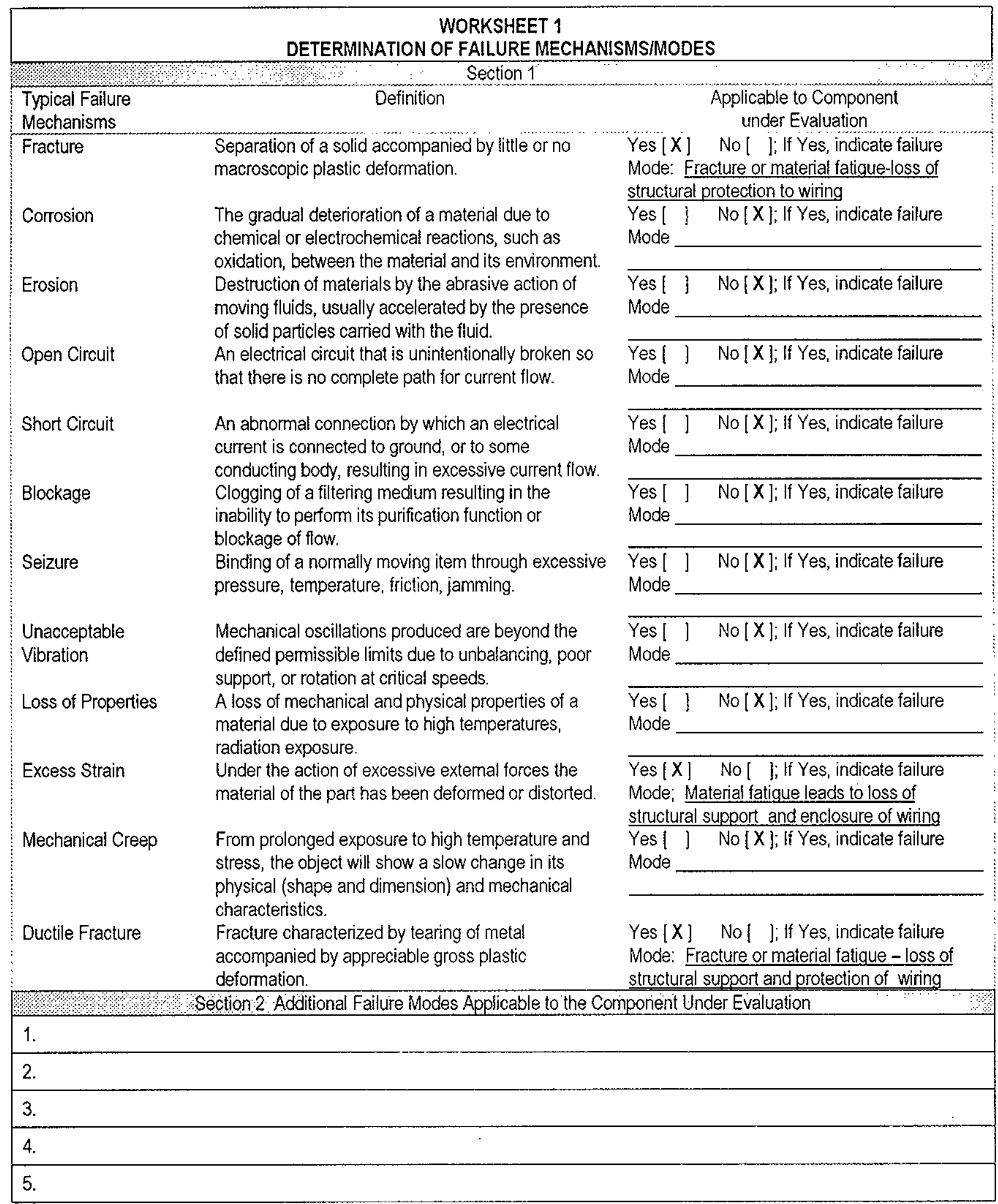




\begin{tabular}{|l|l|}
\hline Commercial Grade Item Upgrade Dedication Form & SNi-4914. Rev. 0 \\
\hline ECN No. NA & Page 12 of 33 \\
Title: CONDUIT FOR SAFETY CLASS WIRING & \\
\hline
\end{tabular}

CHECKLIST 1

ACCEPTANCE METHOD 1

SPECIAL TEST/INSPECTION VERIFICATION

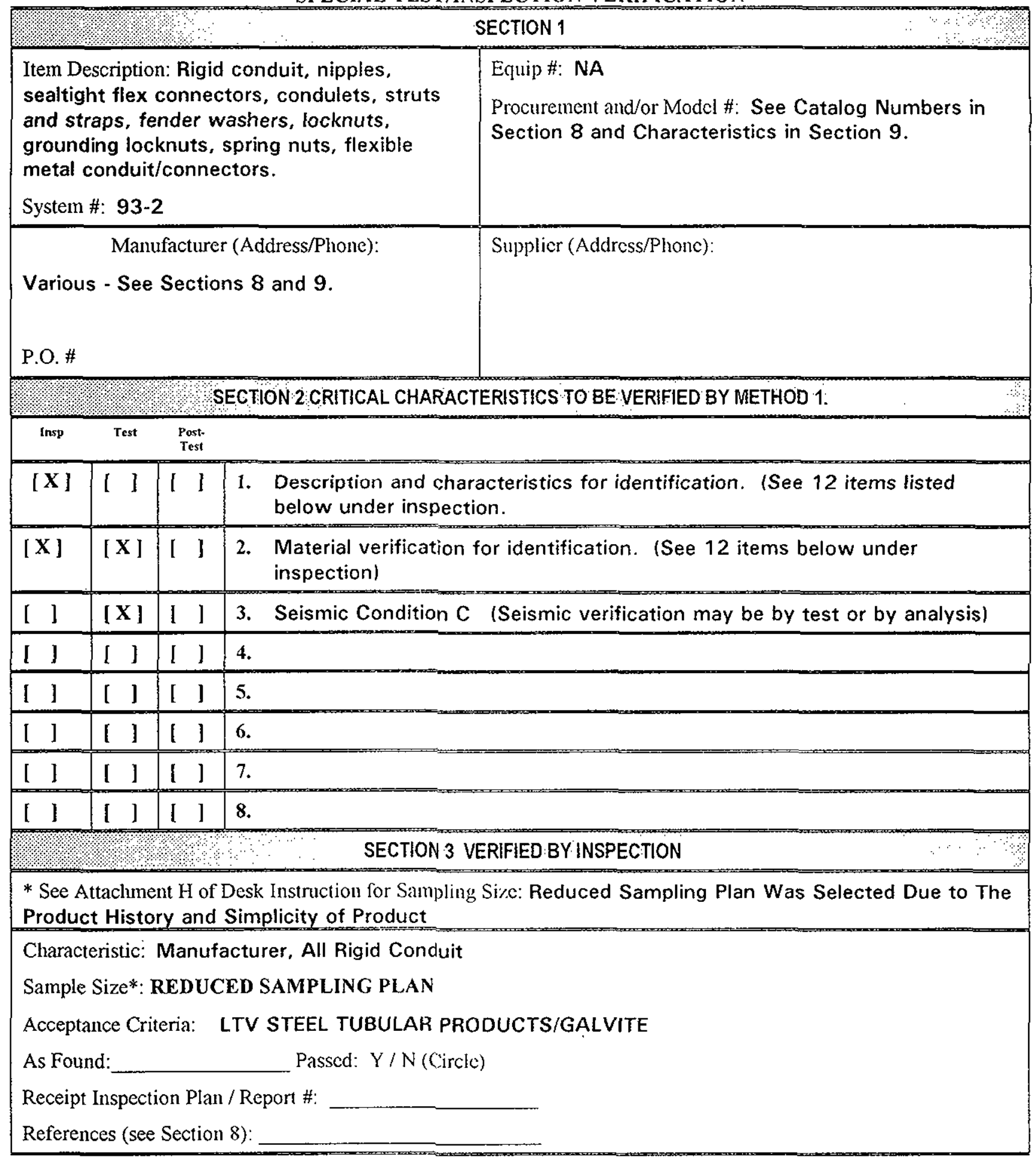


Title: CONDUIT FOR SAFETY CLASS WIRING

\section{Characteristic: All Rigid Conduit}

Sample Size*: REDUCED SAMPLING PLAN

Size / Acceptance Criteria: Verify UL marking on the conduit sample lot.

Verify Size marking on the conduit sample lot or verify identifying dimensions on the conduit sample lot as follows:

$$
\begin{aligned}
& 1 / 2 \text { " / Nominal 0.622 ID } \\
& 3 / 4 " \text { / Nominal } 0.824 \text { ID } \\
& 1 " \text { / Nominal } 1.049 \text { ID } \\
& 11 / 4 " \text { / Nominal } 1.380 \text { ID } \\
& 11 / 2 " \text { / Nominal } 1.610 \text { ID }
\end{aligned}
$$

As Found Passed: $Y / N$ (Circle)

Receipt Inspection Plan / Report \#:

References (see Section 8):

Characteristic: Threaded Rigid Conduit Nipples, Manufacturer

Sample Size*: REDUCED SAMPLING PLAN

Acceptance Criteria: Conduit Pipe Products

As Found: Passed: $\mathrm{Y} / \mathrm{N}($ Circle)

Receipt Inspection Plan / Report \#:

References (see Section 8):

Characteristic: Threaded Rigid Conduit Nipples

Sample Size*: REDUCED SAMPLING PLAN

Size / Acceptance Critcria: Verify UL marking on the sample lot.

Verify Size marking on the sample lot or verify identifying dimensions on the sample lot as follows:

$1 / 2$ " / Nominal 1/2"

3/4" I Nominal 3/4"

1" / Nominal 1"

1 1/2" / Nominal 1 1/2"

As Found: Passed: $Y / N($ Circlc)

Receipt Inspection Plan / Report \#:

References (see Section 8): 


\begin{tabular}{|l|l|}
\hline Commercial Grade Item Upgrade Dedication Form & SNF-4914, Rev. 0 \\
\hline ECN No. NA & Page 14 of 33 \\
Title: CONDUIT FOR SAFETY CLASS WIRING & \\
\hline
\end{tabular}

Characteristic: Threaded Rigid Conduit Bushed Nipples, Manufacturer

Sample Size*: REDUCED SAMPLING PLAN

Acceptance Criteria: Appleton Electric Company

As Found: Passed: Y / N (Circle)

Receipt Inspection Plan / Report \#:

References (see Section 8):

Characteristic: Threaded Rigid Conduit Bushed Nipples

Sample Size*: REDUCED SAMPLING PLAN

ID Number / Acceptance Criteria: Verify UL marking on the sample lot.

Verify part identifying marking on the sample lot or verify identifying Dimensions on the sample lot as follows:

CN-50 / Nominal 1/2"; "A" Dimension, Nominal 7/16"

CN-75/ Nominal 3/4"; "A" Dimension, Nominal 1/2"

CN-100/Nominal 1"; "A" Dimension, Nominal 5/8"

CN-125/ Nominal 1 1/4"; "A" Dimension, Nominal 11/16"

CN-150 / Nominal 1 1/2"; "A" Dimension, Nominal 7/8"

As Found: Passed: $Y / N$ (Circle)

Receipt Inspection Plan / Report \#:

References (see Section 8):

Characteristic: Manufacturer, Threaded Rigid Conduit 3 Piece Connectors

Sample Size*: REDUCED SAMPLING PLAN

Acceptance Criteria: Appleton Electric Company

As Found: Passed: $Y / N$ (Circle)

Receipt Inspection Plan / Report \#:

References (see Section 8): 


\begin{tabular}{|l|l|}
\hline Commercial Grade Item Upgrade Dedication Form & SNF-4914. Rev. 0 \\
\hline $\begin{array}{l}\text { ECN No. NA } \\
\text { Title: CONDUIT FOR SAFETY CLASS WIRING }\end{array}$ & Page 15 of 33 \\
\hline
\end{tabular}

Characteristic: All Threaded Rigid Conduit 3 Piece Connectors

Sample Size*: REDUCED SAMPLING PLAN

ID Number/Acceptance Criteria: Verify UL marking on the sample lot.

Verify identifying marking on the sample lot or identifying dimensions on the sample lot as follows:

EC-50 / Nominal 1/2"; "A' Dimension, Nominal $17 / 16$ "

EC-75 / Nominal 3/4"; "A" Dimension, Nominal 1 9/16"

EC-100/Nominal 1"; "A" Dimension, Nominal $111 / 16$ "

EC-125/Nominal 1 1/4"; "A" Dimension, Nominal $21 / 8$ "

EC-150/ Nominal 1 1/2"; "A" Dimension, Nominal 2 3/16"

As Found: Passed: $Y / N($ Circlc)

Receipt Inspection Plan / Report \#:

References (see Section 8):

Characteristic: Manufacturer, Threaded Rigid Conduit Reducers

Sample Size*: REDUCED SAMPLING PLAN

Acceptance Criteria: Appleton Electric Company

As Found: Passed: $\mathrm{Y} / \mathrm{N}$ (Circle)

Receipt Inspection Plan / Report \#:

References (see Section 8):

Characteristic: All Threaded Rigid Conduit Reducers

Sample Size*: REDUCED SAMPLING PLAN

ID Number/Acceptance Criteria: Verify UL marking on the sample lot.

Verify identifying marking on the parts in the sample lot or verify identifying dimensions on the sample lot as follows:

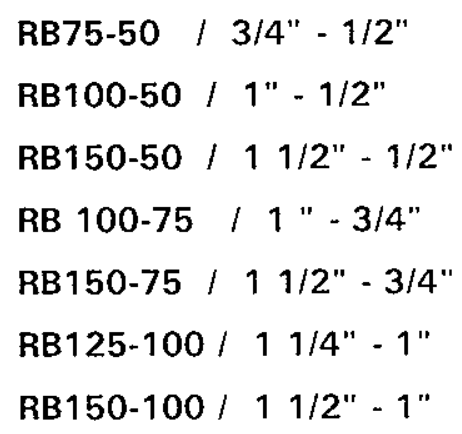

As Found: Passed: $\mathrm{Y} / \mathrm{N}$ (Circle)

Receipt Inspection Plan / Report \#:

References (see Section 8): 


\begin{tabular}{|l|l|}
\hline Commercial Grade Item Upgrade Dedication Form & $S N F-4914$. Rev. 0 \\
\hline $\begin{array}{l}\text { ECN No. NA CGI No. CGI-SNF-D-93-2-C1-052 } \\
\text { Title: CONDUIT FOR SAFETY CLASS WIRING }\end{array}$ & Page 16 of 33 \\
\hline
\end{tabular}

Characteristic: Manufacturer, All Rigid Conduit Condulet Form 35

Sample Size*: REDUCED SAMPLING PLAN

Acceptance Criteria: Appleton Electric Company

As Found: Passed: $\mathrm{Y} / \mathrm{N}$ (Circle)

Receipt Inspection Plan / Report \#:

References (see Section 8):

Characteristic: All Rigid Conduit Condulet Form 35

Sample Size*: REDUCED SAMPLING PLAN

ID Number/Acceptance Criteria: Verify UL marking on the sample lot.

Verify Size marking on the condulet sample lot or verify identifying dimensions on the conduiet sample lot as follows:

LB/T/LR/LL-1/2" / Nominal 1/2"

LB/T/LR/LL-3/4" / Nominal 3/4"

LB/T/LR/LL-1" / Nominal 1"

LB/T/LR/LL-1 1/2" / Nominal 1 1/2"

As Found: Passcd: $\mathrm{Y} / \mathrm{N}$ (Circlc)

Receipt Inspection Plan / Report \#:

References (see Section 8):

Characteristic: Manufacturer, All Rigid Conduit Locknuts And Grounding Locknuts

Sample Size*: REDUCED SAMPLING PLAN

Acceptance Criteria: Appleton Electric Company

As Found: Passed: $\mathrm{Y} / \mathrm{N}$ (Circlc)

Receipt Inspection Plan / Report \#:

References (see Section 8): 


\begin{tabular}{|l|l|}
\hline Commercial Grade Item Upgrade Dedication Form & SNI:-4914. Rev. 0 \\
\hline $\begin{array}{l}\text { ECN No. NA } \\
\text { Title: CONDUIT FOR SAFETY CLASS WIRING }\end{array}$ & Page 17 of 33 \\
\hline
\end{tabular}

\section{Characteristic: All Rigid Conduit Locknuts And Grounding Locknuts}

Sample Size*: REDUCED SAMPLING PLAN

ID Number/Acceptance Critcria: Verify UL marking on the sample lot.

Verify Size marking on the locknut sample lot or verify identifying dimensions on the locknut sample lot as follows:

BL-50, GL-50 / Nominal 1/2"

BL-75, GL-75 / Nominal 3/4"

BL-100, GL-100 / Nominal 1"

BL-125, GL-125 / Nominal 1 1/4"

BL-150, GL-150 / Nominal 1 1/2"

As Found: Passed: $\mathrm{Y} / \mathrm{N}(\mathrm{Circlc})$

Receipt Inspection Plan / Report \#:

References (see Section 8):

Characteristic: Manufacturer, Rigid Conduit Beam Clamps

Sample Size*: REDUCED SAMPLING PLAN

Acceptance Criteria: Appleton Electric Company

As Found: Passed: $\mathrm{Y} / \mathrm{N}$ (Circle)

Receipt Inspection Plan / Report \#:

References (see Section 8):

Characteristic: All Rigid Conduit Beam Clamps

Sample Size*: REDUCED SAMPLING PLAN

ID Number/Acceptance Criteria: Verify identifying marking on the sample lot or verify identifying dimensions on the sample lot as follows:

BH-502 / 2" Size; Jaw Opening Nominal 15/16"

BH-503 / 2 1/2" Size; Jaw Opening Nominal 1 1/16"

As Found: Passcd: $\mathrm{Y} / \mathrm{N}$ (Circlc)

Receipt Inspection Plan / Report \#:

References (see Section 8):

Characteristic: Manufacturer, Liquidtight Flexible Steel Conduit.

Sample Size*: REDUCED SAMPLING PLAN

Acceptance Criteria: Thomas \& Betts

As Found: Passed: $Y / N($ Circle)

Receipt Inspection Plan / Report \#:

References (see Section 8): 


\begin{tabular}{|l|l|}
\hline Commercial Grade Item Upgrade Dedication Form & SNF-4914. Rev. 0 \\
\hline $\begin{array}{l}\text { ECN No. NA CGI No. CGI-SNF-D-93-2-C1-052 } \\
\text { Title: CONDUIT FOR SAFETY CLASS WIRING }\end{array}$ & Page 18 of 33 \\
\hline
\end{tabular}

\section{Characteristic: All Flexible Steel Conduit \\ Sample Size*: REDUCED SAMPLING PLAN \\ ID Number/Acceptance Criteria: Verify UL marking on the conduit sample lot.}

Verify Size marking on the conduit sample lot or verify identifying dimensions on the conduit sample lot as follows:

Type LA-11/ Nominal 1/2" Diameter

Type LA-12/ Nominal 3/4" Diameter

Type LA-13 / Nominal 1" Diameter

Type LA-14/ Nominal 1 1/4" Diameter

Type LA-15/ Nominal $11 / 2 "$ Diameter

As Found: Passed: Y/N (Circle)

Receipt Inspection Plan / Report \#:

References (see Section 8):

Characteristic: Manufacturer, Flexible Metal Conduit Connectors

Sample Size*: REDUCED SAMPLING PLAN

Acceptance Criteria: Thomas and Betts

As Found: Passcd: $Y / N$ (Circle)

Receipt Inspection Plan / Report \#:

References (see Section 8):

Characteristic: All Flexible Metal Conduit Connectors

Sample Size*: REDUCED SAMPLING PLAN

ID Number/Acceptance Criteria: Verify UL marking on the sample lot.

Verify identifying marking on the sample lot or verify identifying dimensions on the sample lot as follows:

5332 / Nominal 1/2" " A" Dimension, Nominal $13 / 8 "$

5333 / Nominal 3/4" -"A" Dimension, Nominal $121 / 32 "$

5334 / Nominal 1" -"A" Dimension, Nominal $17 / 8$ "

5335 / Nominal 1 1/4" "A" Dimension, Nominal 2 9/32"

5336 / Nominal 1 1/2" -A" Dimension, Nominal 2 23/32"

As Found: Passed: $\mathrm{Y} / \mathrm{N}$ (Circle)

Receipt Inspection Plan / Report \#:

References (see Section 8):

Characteristic: Manufacturer, Framing Channels/Pipe Straps

Sample Size*: REDUCED SAMPLING PLAN

Acceptance Criteria: Superstrut

As Found: Passed: $\mathrm{Y} / \mathrm{N}$ (Circlc)

Receipt Inspection Plan / Report \#:

References (see Section 8): 


\begin{tabular}{|l|l|}
\hline Commercial Grade Item Upgrade Dedication Form & SNF-4914. Rev. 0 \\
\hline ECN No. NA CGI No. CGI-SNF-D-93-2-C1-052 & Page 19 of 33 \\
Title: CONDUIT FOR SAFETY CLASS WIRING & \\
\hline
\end{tabular}

\section{Characteristic: All Framing Channels/ Pipe Straps \\ Sample Size*: REDUCED SAMPLING PLAN \\ ID Number/Acceptance Criteria: Half Slot Channel, A-1200-HS / Nominal 1 5/8" x 1 5/8"; 12 gage. or verify identifying dimensions on the sample lot as follows:

$702-1 / 2 /$ Pipe OD Nominal 0.840", 14 gage
$702-3 / 4$ / Pipe OD Nominal 1.050", 14 gage
$702-1 /$ Pipe OD Nominal 1.315", 14 gage
$702-11 / 4$ / Pipe OD Nominal 1.660", 14 gage
$702-11 / 2$ / Pipe OD Nominal 1.900", 14 gage

702 Series Pipe Straps: Verify identifying marking on the sample lot

As Found: Passed: $\mathrm{Y} / \mathrm{N}$ (Circle)

Receipt Inspection Plan / Report \#:

References (see Section 8):

Characteristic: Manufacturer, Fender Washers

Sample Size*: REDUCED SAMPLING PLAN

Acceptance Criteria: $A B C$ Fasteners

As Found: Passed: $\mathrm{Y} / \mathrm{N}$ (Circle)

Receipt Inspection Plan / Report \#:

References (see Section 8):

Characteristic: Fender Washers

Sample Size*: REDUCED SAMPLING PLAN

ID Number/Acceptance Criteria: $20004 / 1 / 4 "$ x 1 1/4"

$20008 / 3 / 8^{\prime \prime} \times 11 / 2^{\prime \prime}$

$20015 / 1 / 2 " \times 2 "$

As Found: Passed: $Y / N($ Circle $)$

Receipt Inspection Plan / Report \#:

References (see Section 8):

Characteristic: Manufacturer, Fasteners

Sample Size*: REDUCED SAMPLING PLAN

Acceptance Criteria: Powerstrut

As Found: Passed: $Y / N($ Circlc)

Receipt Inspection Plan / Report \#:

References (see Section 8): 
Commercial Grade Item Upgrade Dedication Form

ECN No. NA

CGI No. CGI-SNF-D-93-2-C1-052

SNF-4914, Rev. 0

Title: CONDUIT FOR SAFETY CLASS WIRING

Characteristic: Fasteners

Sample Size*: REDUCED SAMPLING PLAN

ID Number/Acceptance Criteria: PS 146 Continuous Threaded Rod / Nominal 3/8"

/ Nominal 1/2"

/ Nominal 5/8"

/ Nominal 3/4"

PS RS Clamping Nut with Regular Spring / 1/4" x 20

$$
\begin{aligned}
& / 3 / 8 " \times 16 \\
& / 1 / 2 " \times 13 \\
& / 5 / 8 " \times 11 \\
& / 3 / 4^{\prime \prime} \times 10 \\
& / 7 / 8^{\prime \prime} \times 9
\end{aligned}
$$

As Found:

Passed: $Y / N($ Circle $)$

Receipt Inspection Plan / Report \#:

References (see Section 8):

Characteristic: Material Identification, All Items (11 items from Section 9-2)

Sample Size*: REDUCED SAMPLING PLAN

Acceptance Criteria: Steel

As Found: Passed: $Y / N($ Circlc $)$

Receipt Inspection Plan / Report \#:

References (see Section 7):

Characteristic: Material Identification, Flexible Steel Conduit Jacket

Sample Size*: REDUCED SAMPLING PLAN

Acceptance Criteria: Flexible PVC Compound

As Found:

Passed: $\mathrm{Y} / \mathrm{N}$ (Circlc)

Receipt Inspection Plan / Report \#:

References (see Section 8):

Characteristic: Material Identification, Fender Washers

Sample Size*: REDUCED SAMPLING PLAN

Acceptance Criteria: Zinc Coated Steel

As Found: Passed: $\mathrm{Y} / \mathrm{N}$ (Circle)

Receipt Inspection Plan / Report \#:

References (see Section 8): 


\begin{tabular}{|l|l|}
\hline Commercial Grade Item Upgrade Dedication Form & SNF-4914. Rov.0 \\
\hline $\begin{array}{l}\text { ECN No. NA } \\
\text { Title: CONDUIT FOR SAFETY CLASS WIRING }\end{array}$ & Page 21 of 33 \\
\hline
\end{tabular}

\section{SECTION 4 VERIFIED BY SPECIALTEST}

* See Attachment $H$ of Desk Instruction for Sampling Size

Test To Be Performed by:

I ] Purchaser

[ ] Supplier/Manufacturer**

[ ] Other

Number of Itcms to be Testcd: Reduced Sampling Plan Was Selected Due To The Product History and Simplicity Of Product

Test/Inspection Location:

Characteristic for Test: Seismic Condition C - All Items (13 items from Section 9-2)

Acceptance Criteria: Maintain Critical Function Before, During, and After Seismic Event.

Sample Size*: Sample Size is NA because seismic verification of the installed conduit system with fittings, supports, etc, will be done by analysis. Physical testing is not anticipated.

Actual Test Value:

Test Plan and Report \#: References (sce Section 8):

Characteristic for Test:

Acceptance Criteria:

Sample Size*: REDUCED SAMPLING PLAN

Actual Test Value:

Test Plan and Report \#: References (sec Section 8):

Characteristic for Test:

Acceptance Criteria:

Sample Size*: REDUCED SAMPLING PLAN

Actual Test Value:

Test Plan and Report \#: References (sce Scction 8):

** If Supplier/Manufacturer or Other. Refer to C(i] Checklist-2 for Support lnformation 


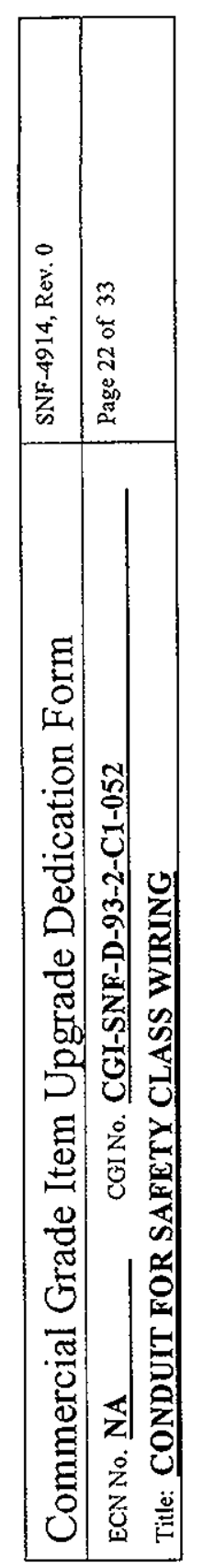

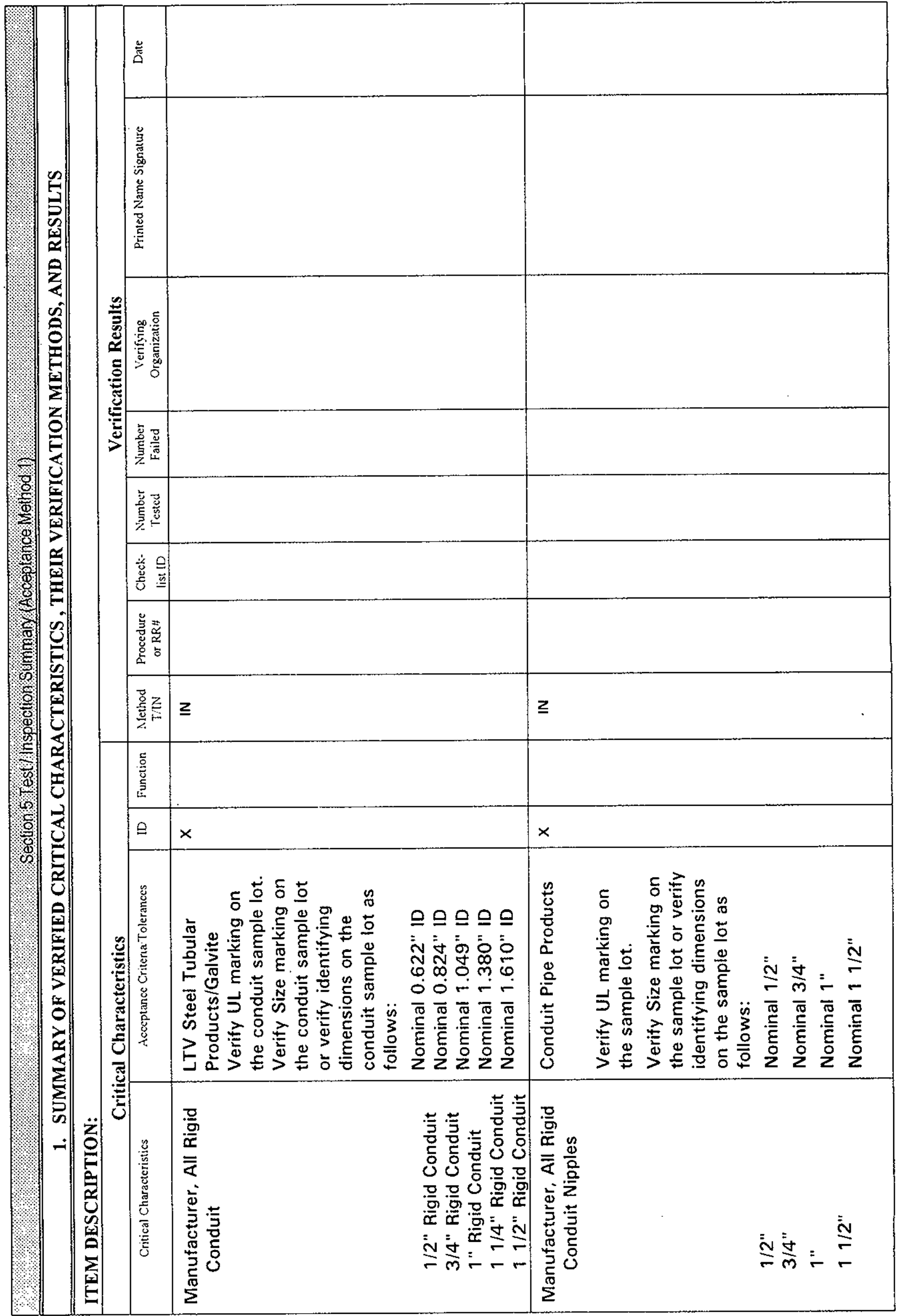




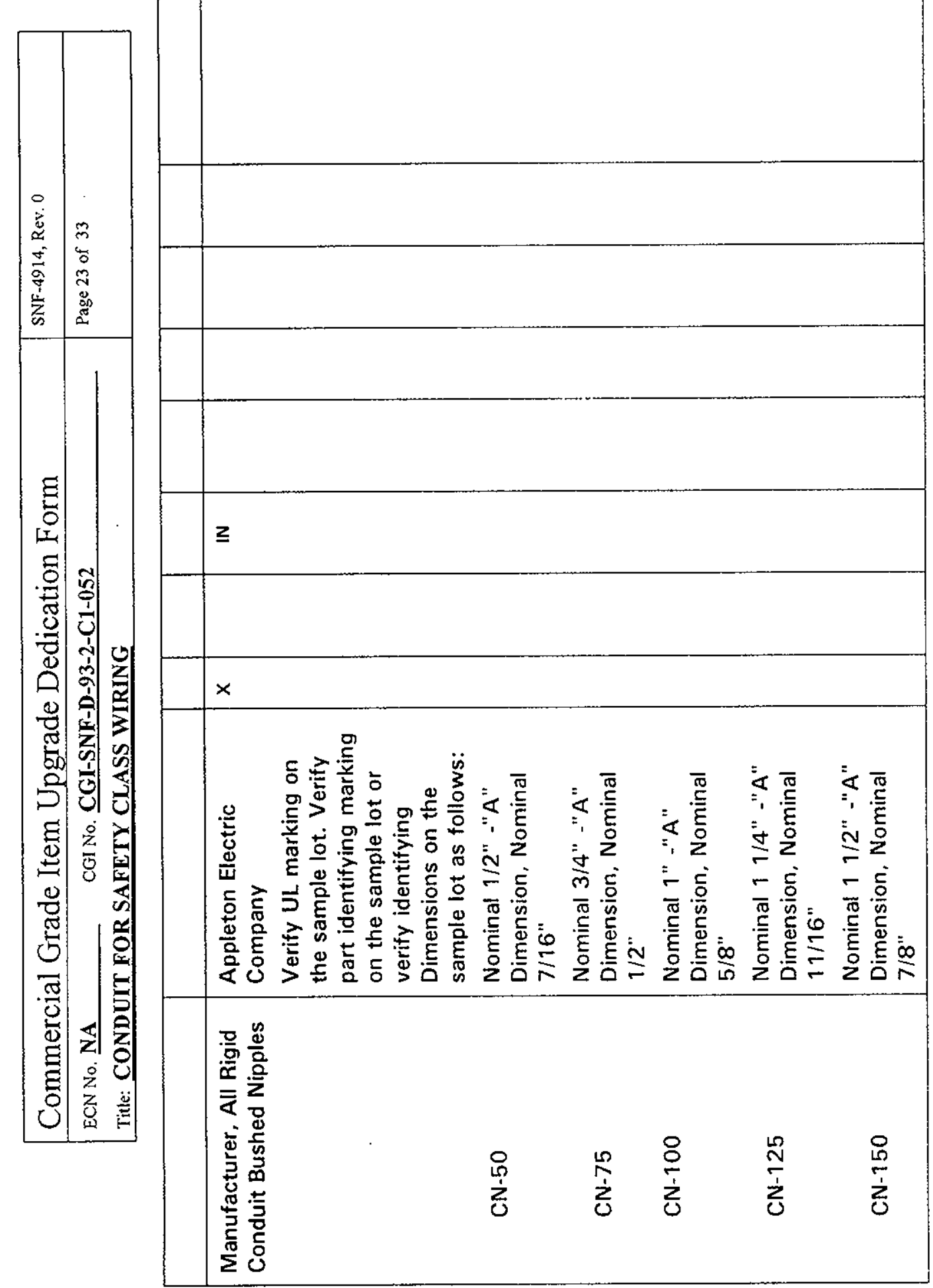




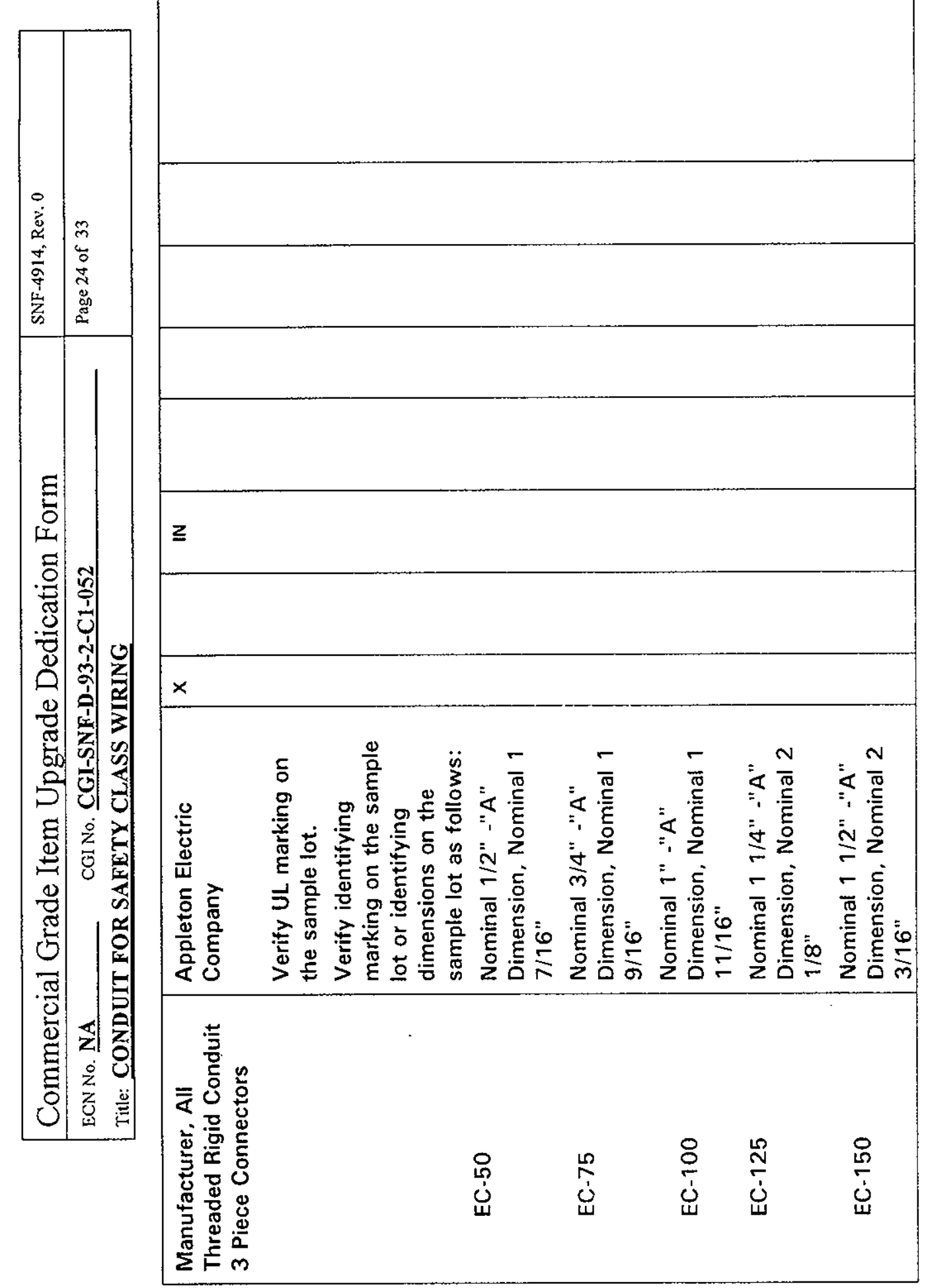




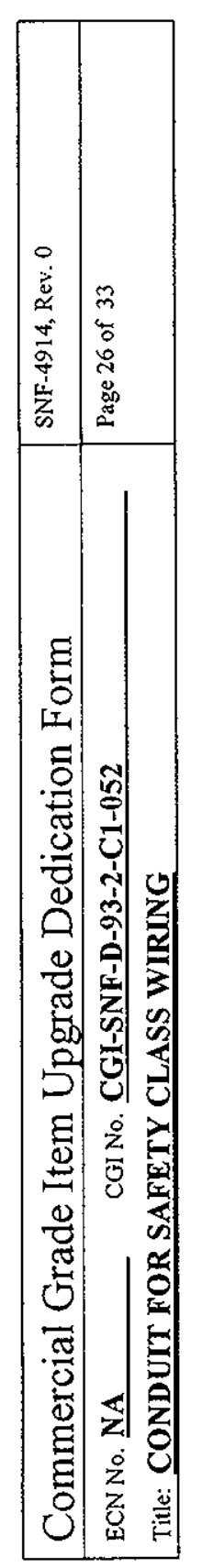

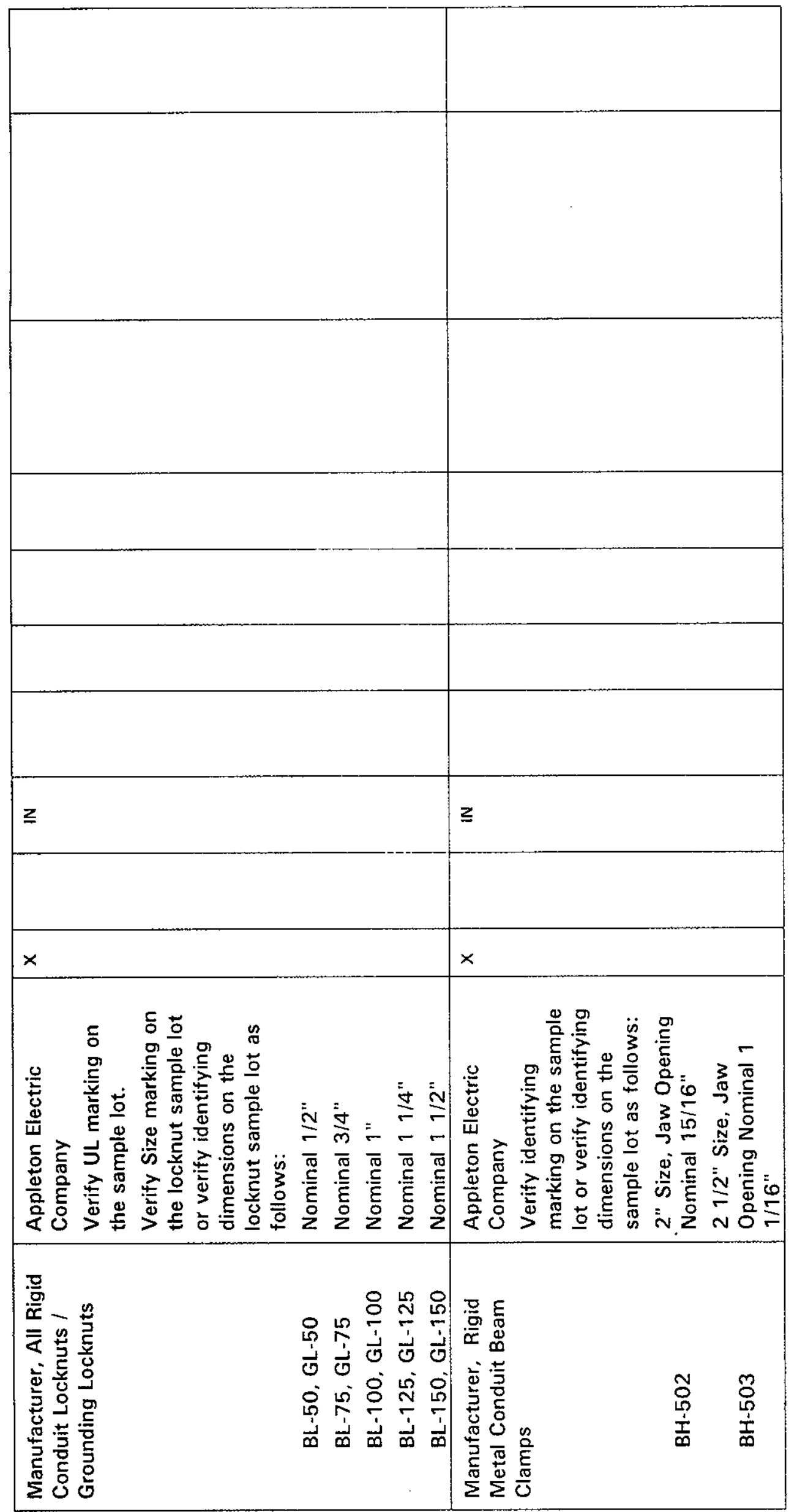




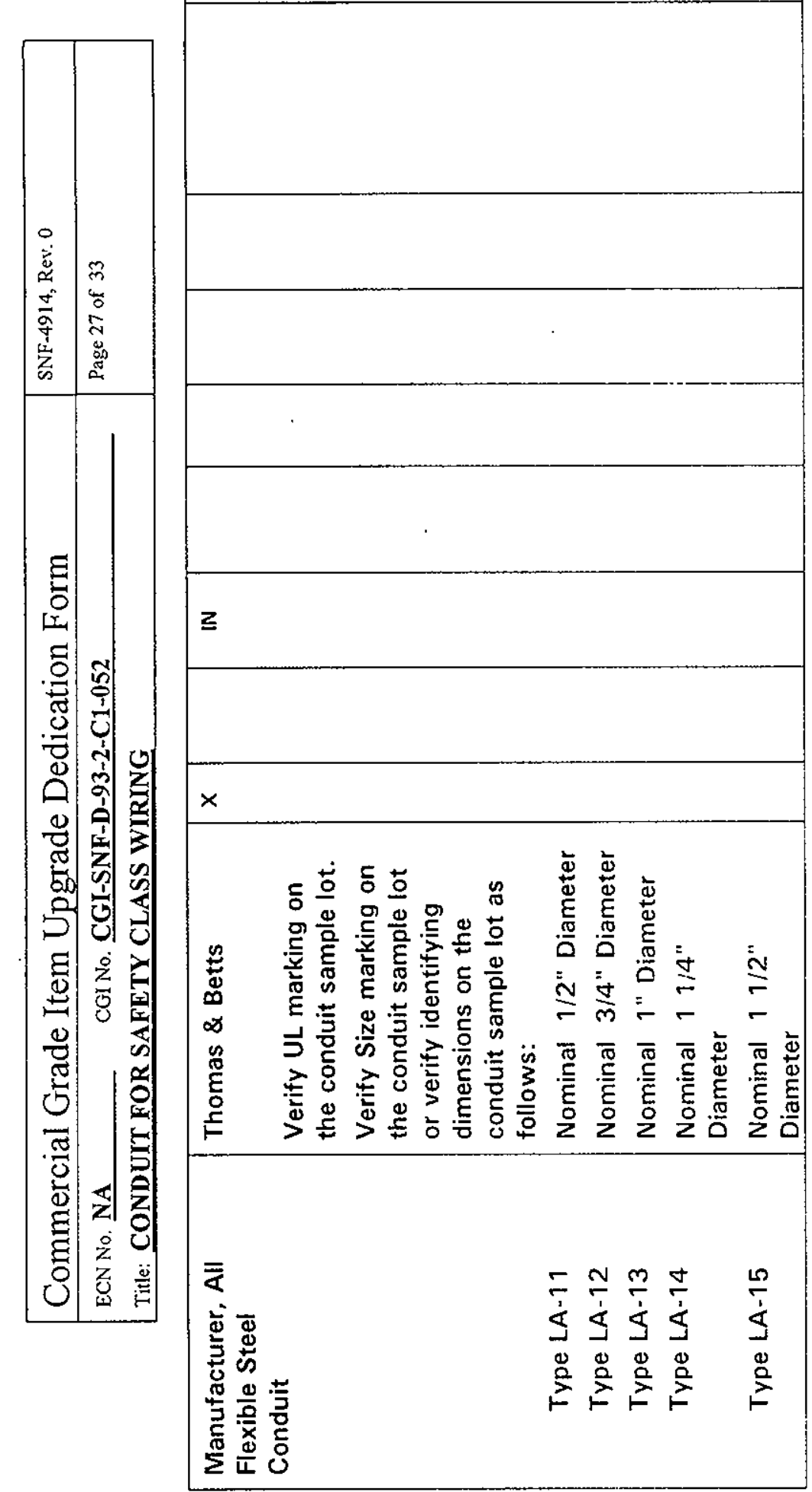




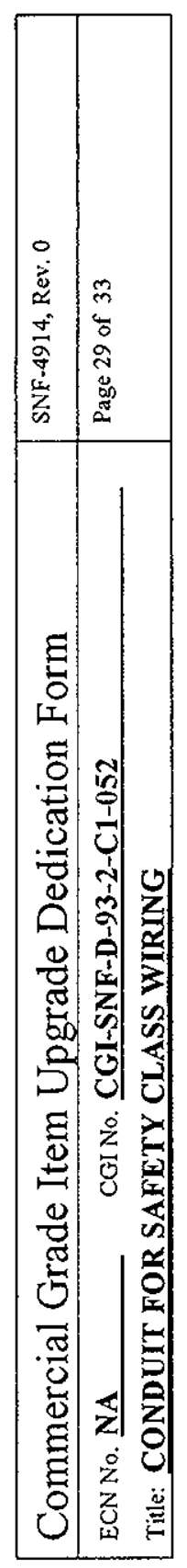

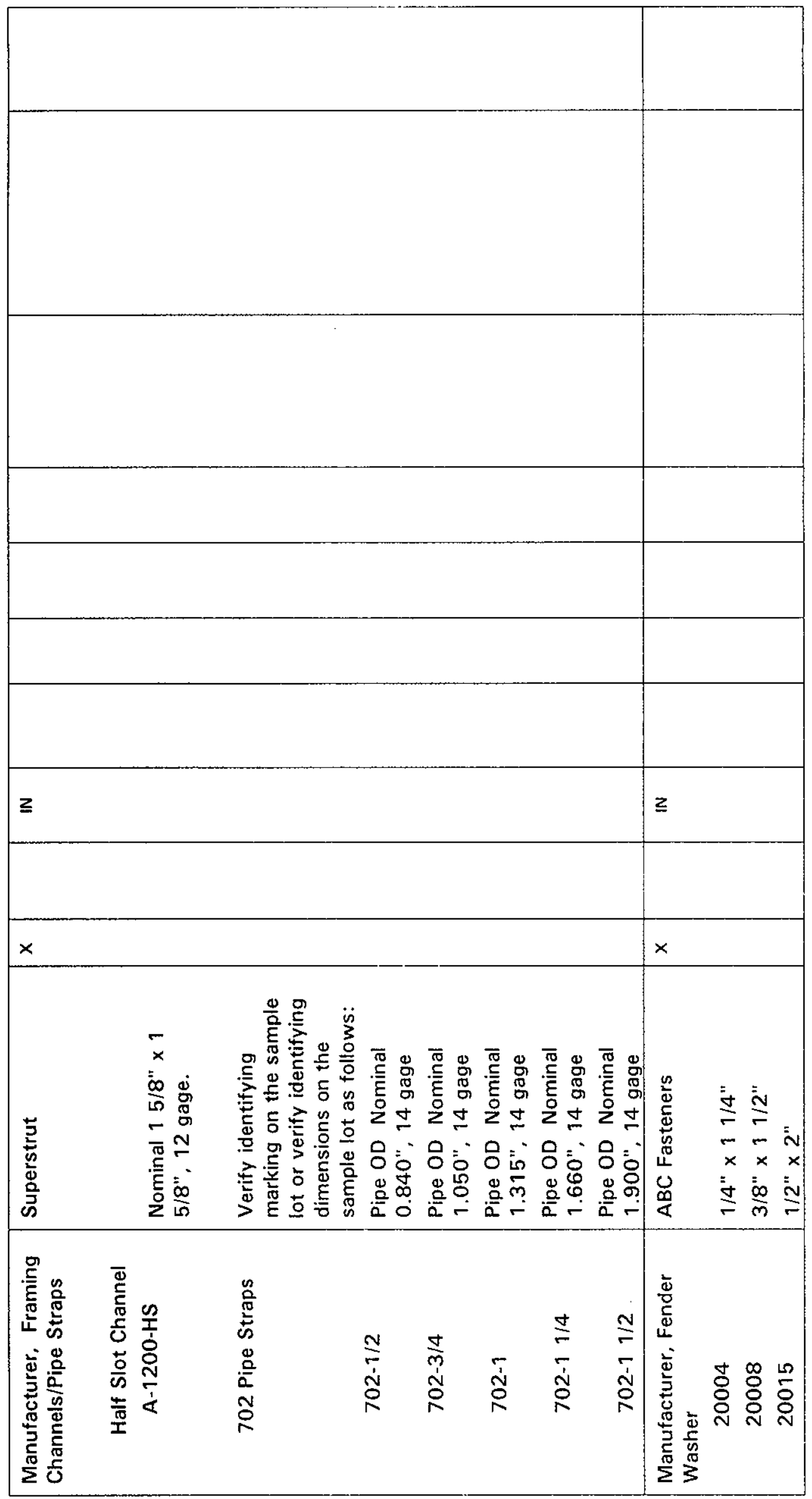




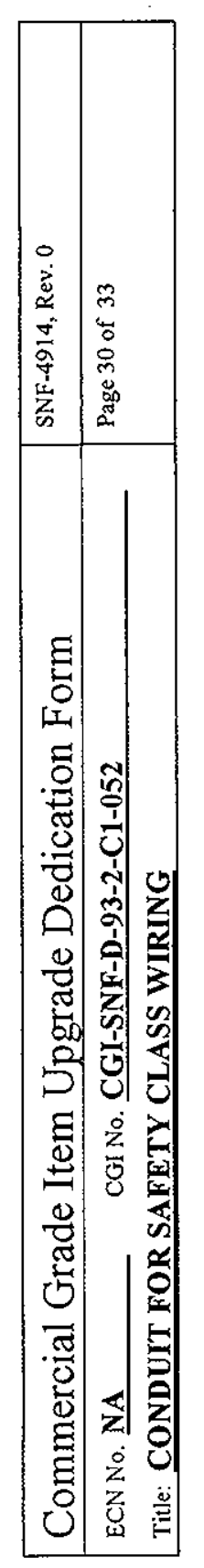

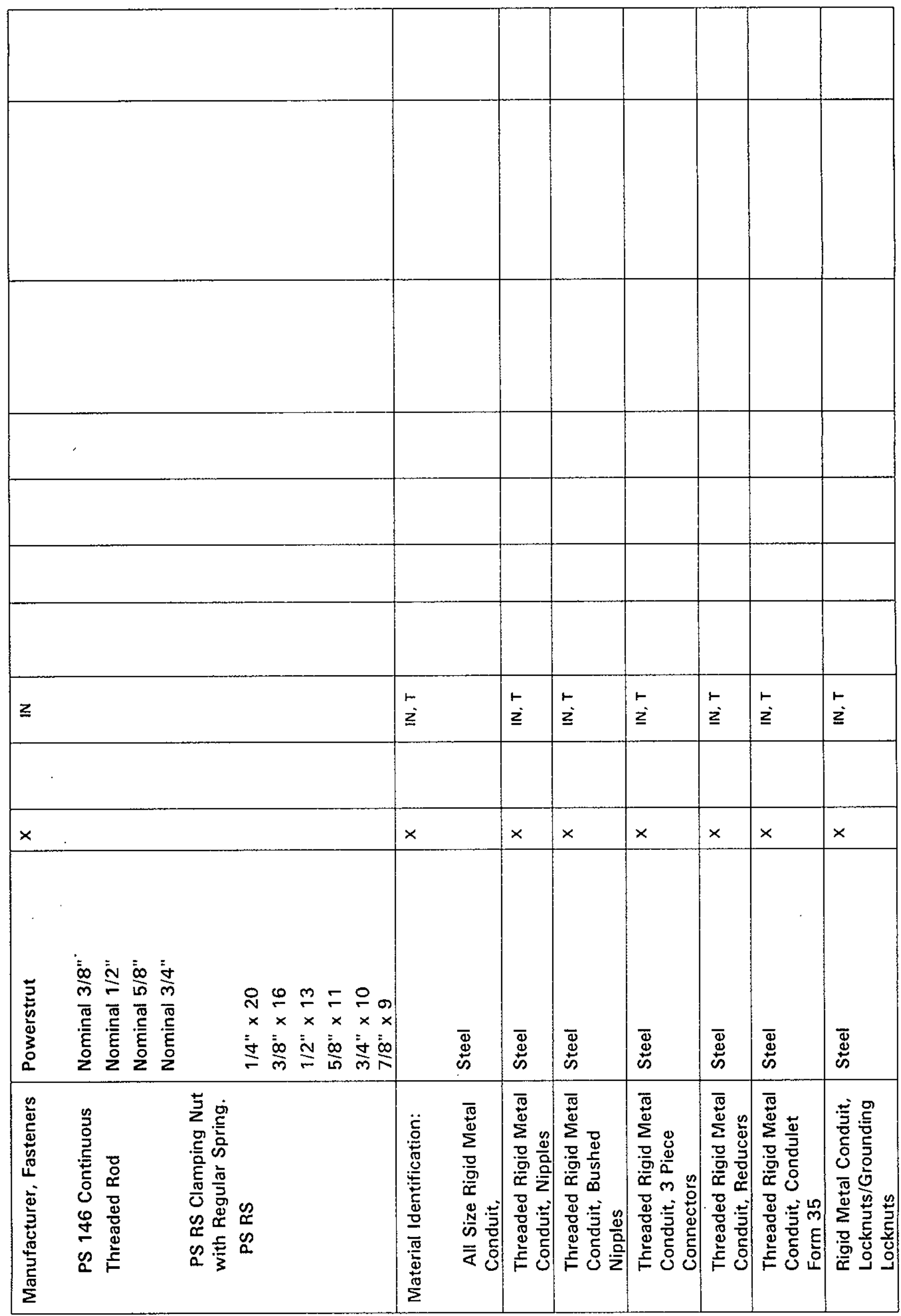




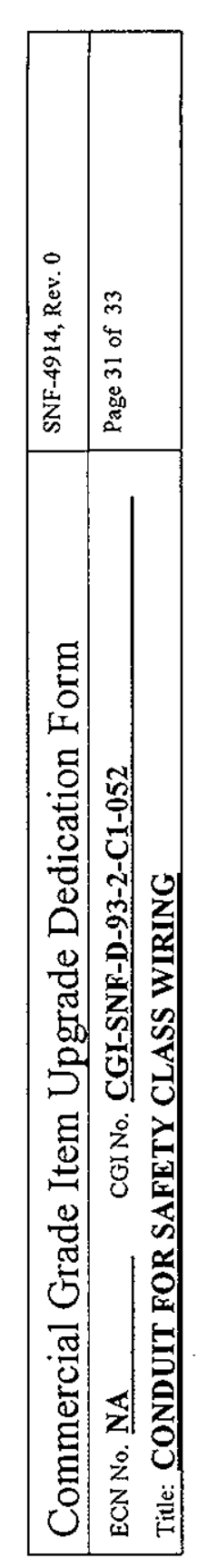

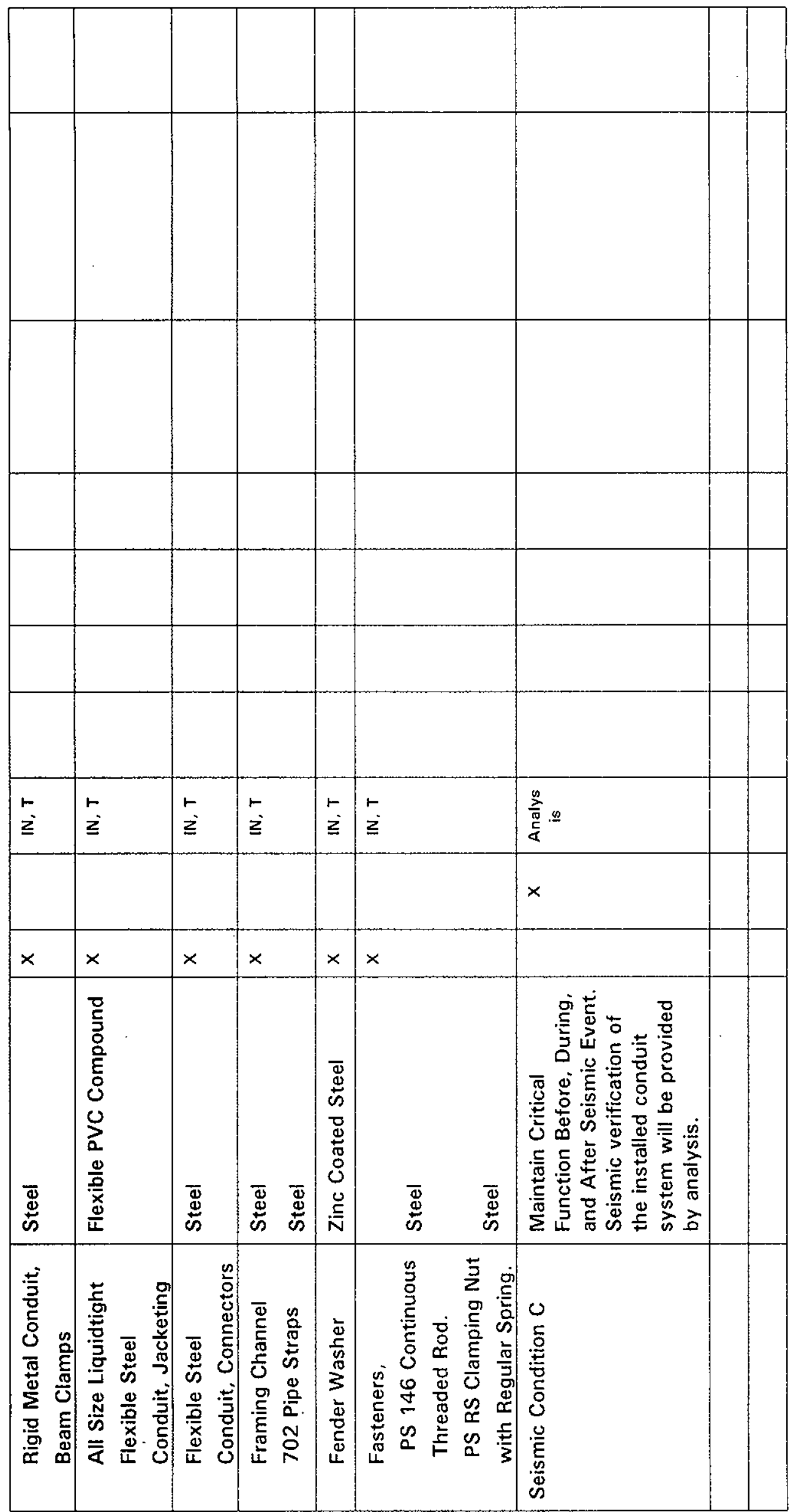




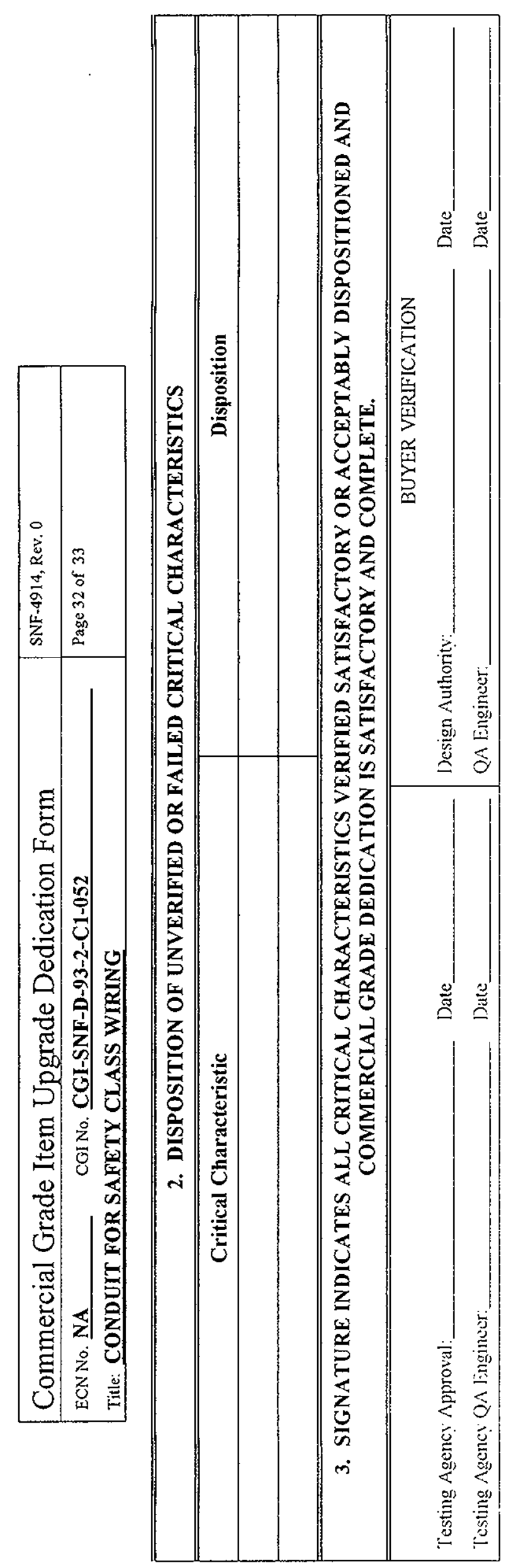




\begin{tabular}{|l|l|}
\hline Commercial Grade Item Upgrade Dedication Form & S.Ni-4914. Rev. 0 \\
\hline $\begin{array}{l}\text { ECN No. NA } \\
\text { Title: CONDUIT FOR SAFETY CLASS WIRING }\end{array}$ & Page 33 of 33 \\
\hline
\end{tabular}

\begin{tabular}{|c|c|c|c|}
\hline \multicolumn{3}{|l|}{ Name } & \multirow[t]{2}{*}{ Phone } \\
\hline Design Authority & ( & ) & \\
\hline QA & ( & ) & \\
\hline QC & ( & ) & \\
\hline Cog - Engineèr & ( & ) & \\
\hline CGI Engineer & ( & ) & · \\
\hline Procurement Engineer & ( & ) & \\
\hline Other & ( & ) & \\
\hline , & ENTATION FOR THIS C & ECKLLIST & \\
\hline Initial Procurement Documents & For Critical Charac & ristics & \\
\hline [ ] Drawings: & & & \\
\hline [ ] Manuals (specify type \& number): & & & \\
\hline [ ] Design Calculations & & & \\
\hline [ ] Installation Instructions & & & \\
\hline [ ] Operation Instructions & & & \\
\hline [ ] Calibration Instructions & & & \\
\hline [ ] Manufacturer's Recommended Spare Parts List & & & \\
\hline [ ] Other: & & & \\
\hline Procurement Documents & & & \\
\hline [ ] Certificate of Conformance/Compliance & & & \\
\hline [ ] Seismic Qualification Certificate & & & \\
\hline [ ] Environmental Qualification Certificatc & & & \\
\hline [ ] Test Report (s): & & & \\
\hline [ ] Inspection Report (s): & & & \\
\hline [ ] CMTRs for ASME Pressure Retaining Materials & & & \\
\hline [ ] Valve Seat Leakage Report & & & \\
\hline [ ] Weld Records & & & \\
\hline [ ] Material Traceability Record & . & & \\
\hline [ ] Other: & & & \\
\hline
\end{tabular}




\section{DISTRIBUTION SHEET}

\begin{tabular}{|l|l|}
\hline To & From \\
Distribution & SNE-CVD \\
\hline Project Titemonk Order
\end{tabular}

Project Title $/$ Work Order

W-441, SNF-4914, CONDUIT FOR SC WIRING

Name

C. Miska/D. Whitehurst

C. Van Katwijk

K. Boes (CVD Library)

R. Ramsgate

J. Brehm

D. Whitworth

P. Morrel? (AVS)

M. Evarts (AI)

D. Marburger

SNE startup
Page 1 of 1

Date $7 / 28 / 99$

EDT No. 626324

ECN No. N/A

\begin{tabular}{c|c|c|c|c}
\hline MSIN & $\begin{array}{c}\text { Text } \\
\text { With All } \\
\text { Attach. }\end{array}$ & Text Only & $\begin{array}{c}\text { Attach./ } \\
\text { Appendix } \\
\text { Only }\end{array}$ & $\begin{array}{c}\text { EDT/ECN } \\
\text { Only }\end{array}$ \\
\hline
\end{tabular}

